\title{
LATTICE DISORDERING, PHASE TRANSITION, AND SUBSTRATE TEMPERATURE EFFECTS IN MEV-ION-IMPLANTED III-V COMPOUND SEMICONDUCTORS
}

\author{
Fulin Xiong ${ }^{*}$, C. J. Tsai ${ }^{\dagger}$, T. Vreeland Jr. ${ }^{\dagger}$, and T. A. Tombrello ${ }^{\dagger}$ \\ *Gordon McKay Laboratory of Applied Science, Harvard University, Cambridge, MA 02138 \\ California Institute of Technology, Pasadena, CA 91125
}

\begin{abstract}
An experimental study of lattice disordering, the crystalline-to-amorphous (c-a) phase transition, and substrate temperature effects in $\mathrm{MeV}$-ion-implanted III-V compound semiconductor crystals is presented. A comparison has been made between the GaAs and InP systems, which have been implanted with $2 \mathrm{MeV}$ oxygen ions at either room temperature (RT) or near liquid nitrogen temperature (LT). A strong in situ dynamic annealing has been found in the RT implanted GaAs, and the LT implanted GaAs exhibits heterogeneous (at the end-of-range of the ions) and homogeneous (at the subsurface region) c-a phase transitions. In InP crystals, in situ annealing is much less pronounced in RT implantation, and dose-dependent damage nucleation and layer-by-layer amorphization take place. LT implantation results in lattice disordering and phase transition with a critical dose at least one order lower than that for GaAs. The mechanisms and kinetics of lattice disordering by ion irradiation are also discussed.
\end{abstract}

\section{INTRODUCTION}

$\mathrm{MeV}$ ion implantation into III-V compound semiconductors has attracted considerable attention in recent years due to its application potential for modifying deeply buried layers and for fabricating 3-dimensional devices $[1,2]$. An understanding of the mechanisms for lattice disordering and its associated physical phenomena during $\mathrm{MeV}$ ion implantation is of technological importance and scientific interest. With respect to the ionsolid interaction, extension of the ion energy to the $\mathrm{MeV}$ range provides many advantages (such as deep implantation and minimized surface damage), but it also leads the ion-solid interaction into a new regime where many associated effects need to be understood. In the material aspects, differing from the damage processes in single elemental semiconductors, defect generation under ion implantation in compound semiconductors is rather complicated due to their binary nature and defect complexity. In addition, the conditions for implantation, such as the substrate temperature and the ion beam flux density are also important in determining the final state of the damage profile and the dopant distribution.

In this paper, an experimental study of lattice disordering, the crystalline-toamorphous (c-a) phase transition, and substrate temperature effects in $2 \mathrm{MeV}$-oxygen-ionimplanted III-V compound semiconductor crystals is presented. A comparison has been made between the GaAs and InP systems with the substrates at either room temperature or near liquid nitrogen temperature. The results have revealed marked differences in terms of lattice disordering, lattice strain profiling, and threshold doses for the c-a transition in two materials as well as temperature influence. Mechanisms and kinetics of lattice disordering by ion irradiation in compound semiconductors are also discussed.

\section{EXPERIMENTAL ASPECTS}

Commercially available n-type $\mathrm{GaAs}(100)$ and $\mathrm{InP}(100)$ single crystal substrates were implanted by $2 \mathrm{MeV}$ oxygen ions generated from the Caltech Tandem Accelerator over a broad dose range. The implantation was carried out with the target holder maintained either at ambient room temperature (RT) or at low temperature (LT) with liquid nitrogen cooling (about $100 \mathrm{~K}$ on the target surface). The focused and electrostatically rastered beam was projected onto the sample surface in a non-channeling direction through a $6 \times 6 \mathrm{~mm}^{2}$ collimator. The beam current density in all cases was maintained constant at about $2.5 \times 10^{12}$ ions $/ \mathrm{cm}^{2} \mathrm{sec}$.

Sample characterization was done at $\mathrm{RT}$ using the $\mathrm{x}$-ray rocking curve technique (XRC) for measuring lattice strain ${ }^{[3]}$ and high energy ion channeling for profiling crystalline 
damage and the defect distribution ${ }^{[4]}$. Based on Bragg diffraction, the XRC gives a measure of the change of $x$-ray diffraction intensity (reflecting power) vs. angle in the vicinity of a Bragg peak due to lattice strain and crystal imperfections. Symmetrical (400) diffraction was used for perpendicular strain measurement. The profiles of lattice strain and the crystalline damage parameter (which accounts for the standard deviation of a Gaussian distribution of atomic displacement of lattice atoms from their regular sites) were obtained by deconvolution of experimental XRCs with the dynamical diffraction model[5,6]. Channeling RBS (CRBS) measurements were carried out either at the Caltech Tandem Accelerator Laboratory (for GaAs) with $4 \mathrm{MeV}$ He ions and a detection angle of $140^{\circ}$, or at the Laboratory of Cambridge Accelerator for Materials Science (for InP) with $3 \mathrm{MeV}$ He ions and a detection angle at $175^{\circ}$. The beam was incident on the sample along the surface normal for channeling measurements and $7^{\circ}$ off for random spectra. A quantitative estimation of lattice disordering induced by ion implantation can be derived from the net dechanneling yield $\chi$, defined as $\chi=\left(C_{i}-C_{0}\right) /\left(C_{r}-C_{0}\right)$, where $C_{i}, C_{0}$, and $C_{r}$ are dechanneling yields of an implanted sample, a virgin sample, and a random spectrum.

\section{RESULTS}

Two sets of channeling spectra from RT and LT implanted GaAs samples are plotted in figure 1 . There exist two distinct damaged regions: the heavy damaged region, ranging from $1 \mu \mathrm{m}$ to $2 \mu \mathrm{m}$ deep below the surface, and the light damaged region ranging within the first $1 \mu \mathrm{m}$ near the surface. Appearance of these two regions is characteristic of $\mathrm{MeV}$ ion implantation, differing from low energy $(\mathrm{keV})$ ion implantation where the heavy damage region dominates in the whole implanted surface layer. Fig. 1 illustrates clearly the evolution of lattice disorder in these two regions as a function of the implantation dose, as well as the effect of the influence of temperature.Lattice disorder increases markedly with the implantation dose, especially in the LT implanted samples. Figure 2 plots the net dechanneling yields as a function of the dose within the highly disordered region as indicated in Fig. 1. In the low dose regime $\left(10^{13}\right.$ to $\left.10^{14} / \mathrm{cm}^{2}\right)$, lattice damage accumulates with dose,

$\mathrm{D}$, as $\chi=c D^{\mathrm{n}}$, with $\mathrm{n}$ having a value around 1. This indicates that the lattice disordering in the low dose regime increases linearly with the dose with little damage overlap and defect recombination. In the high dose regime, the lattice damage reaches a saturation state, differing at the different temperatures. In LT implantation (Fig. la), lattice disorder saturated at $100 \%$, indicating heterogenous amorphization taking place with threshold dose around $1 \times 10^{15}$ ions $/ \mathrm{cm}^{2}$. The thickness of the amorphous layer
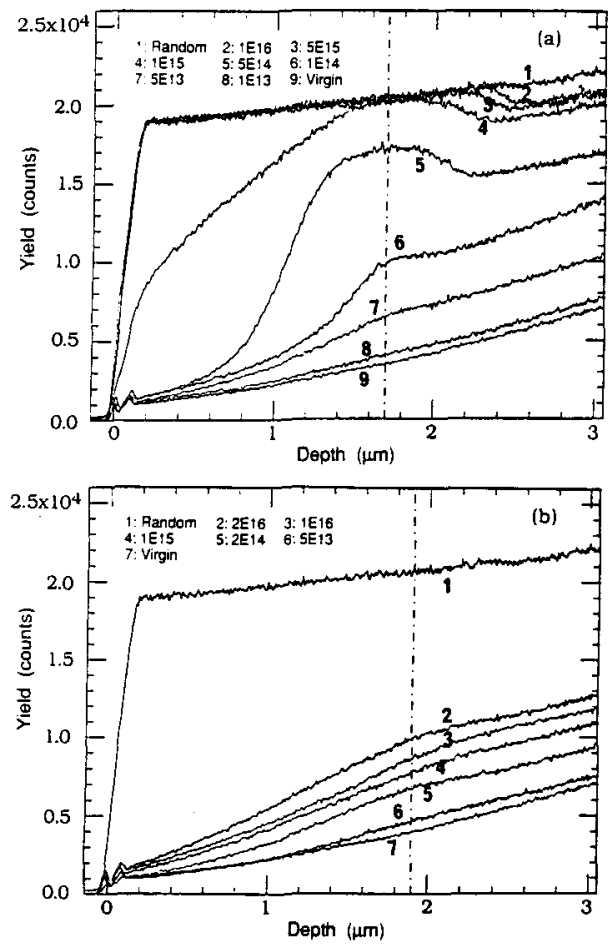

Figure 1. Channeling RBS spectra from GaAs implanted with $2 \mathrm{MeV}$ oxygen ions (a) LT and (b) at RT with doses marked. 
increases as the dose increases. In the RT implantation (Fig. 1b), lattice disorder is much less pronounced, with a saturation level at about $30 \%$. This indicates that strong in-situ annealing takes place during implantation. As a result, only a small amount of lattice disorder is sustained after implantation. No c-a transition was observed at doses up to $2 \times 10^{16}$ ions $/ \mathrm{cm}^{2}$. Such an in situ annealing effect has also been observed in $\mathrm{MeV}$ $\mathrm{Si}$ ion implantation[7]. In the subsurface region, lattice damage accumulates very slowly with increasing dose since nuclear damage is minimized in this region. However, for LT implantation, the lattice damage accumulates quick enough so that a homogeneous $\mathrm{c}-\mathrm{a}$ transition takes place with a threshold dose at about $5 \times 10^{15}$ ions $/ \mathrm{cm}^{2}$. While for RT implantation, this region maintains minimal disorder .

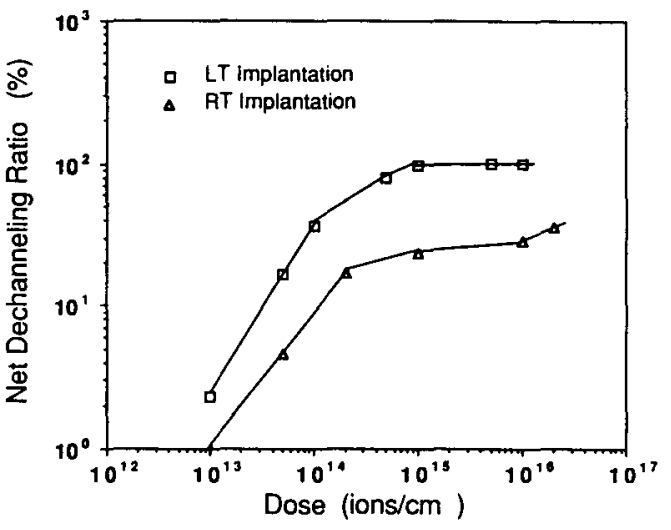

Figure 2. The net dechanneling ratio in the heavy damage region as a function of the ion dose in $2 \mathrm{MeV}$ oxygen ion implanted GaAs. The data are taken from figure 1 .

$\mathrm{MeV}$ ion implantation into InP exhibits markedly different features from GaAs in terms of dose-dependent evolution of damage production and amorphization. Figure 3 shows two sets of CRBS spectra from InP samples. Though two distinct damaged regions are still clearly distinguishable at low doses with the surface region having minimal lattice disorder, production of lattice disorder under the same implantation condition is much more efficient in InP than in GaAs. At LT implantation (Fig. 3a), the threshold for amorphization is around $5 \times 10^{13}$ ions $/ \mathrm{cm}^{2}$, one order of magnitude lower than in the case of GaAs. The entire implanted area goes quickly through the c-a transition at a dose of $2 \times 10^{14} \mathrm{ions} / \mathrm{cm}^{2}$. In RT implantation (Fig. $3 \mathrm{~b}$ ), the in situ self-annealing becomes much less pronounced. In contrast to $\mathrm{GaAs}$, the ion implantation induced $\mathrm{c}$-a transition in InP takes place at a dose of $5 \times 10^{14}$ ions $/ \mathrm{cm}^{2}$. The RBS dechanneling yield saturation extends towards the surface as the ion dose increases, indicating the amorphous region proceeds a layer-by-layer growth.
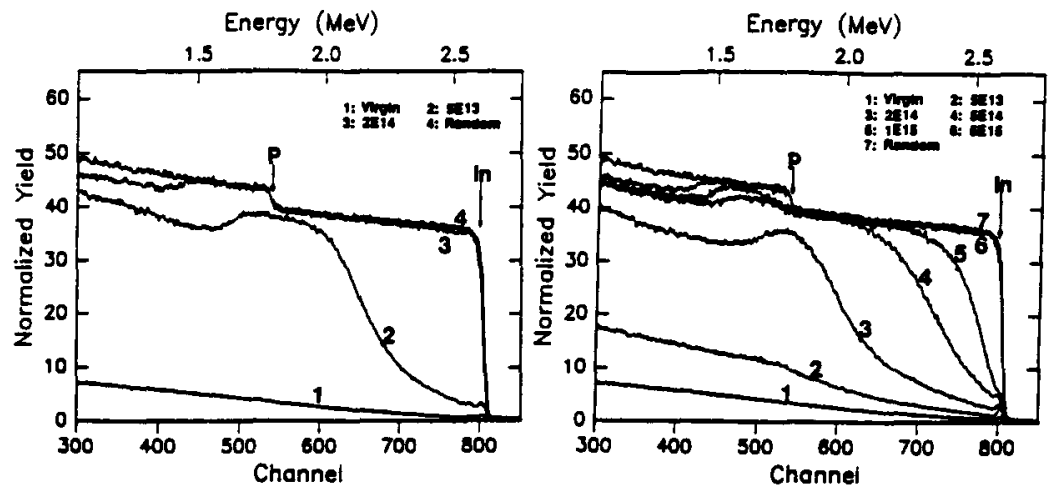

Figure 3. Channeling RBS Spectra from $2 \mathrm{MeV}$-oxygen-ion-implanted InP:

(a) LT implanted and (b) RT implanted, with doses marked. 

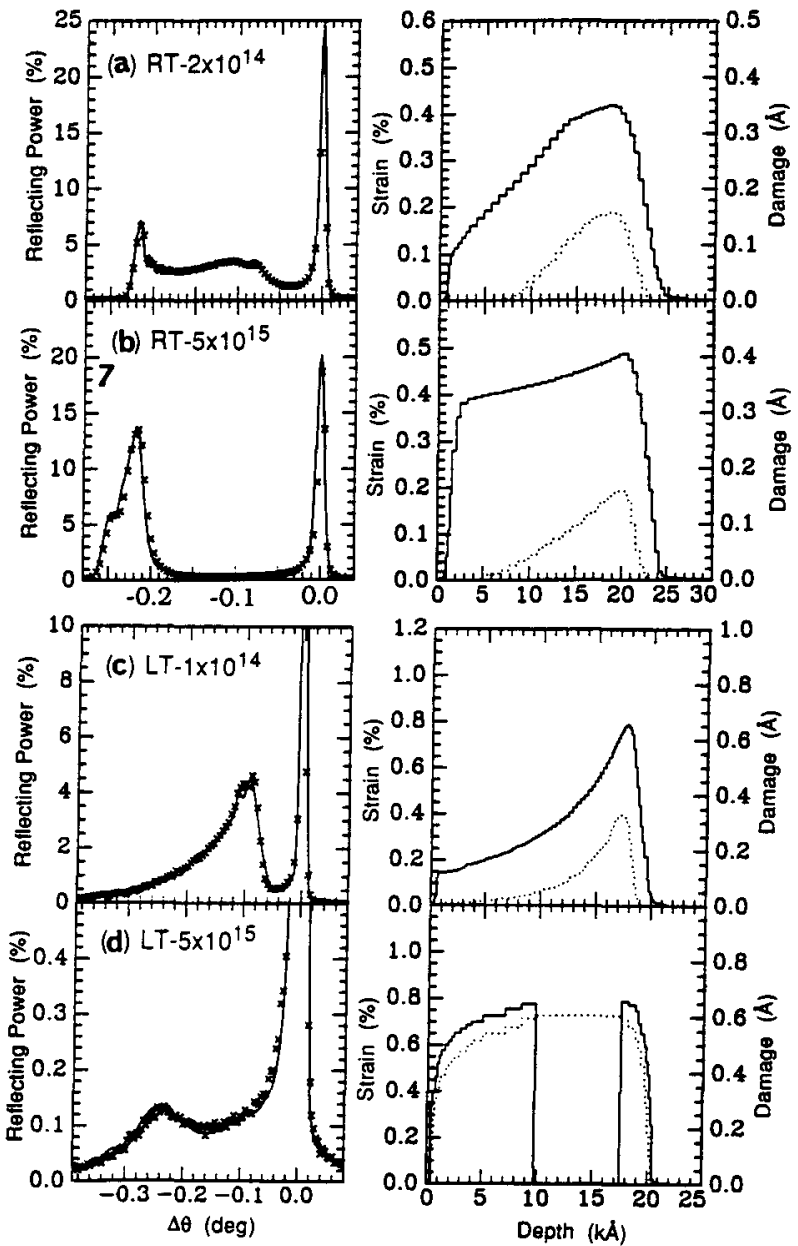

Figure 4. $X$-ray rocking curves (on the left,) and strain profiles (on the right) of 2-MeV-oxygen-ion- implanted $\mathrm{GaAs}$ with ion doses of (a) $2 \times 10^{14}$ and (b) $5 \times 10^{15}$ ions $/ \mathrm{cm}^{2}$ at RT, (c) $1 \times 10^{14}$ and (d) $5 \times 10^{15}$ ions $/ \mathrm{cm}^{2}$ at LT.h On the left: the cross points are experimental and the solid curves are calculated. On the right: the solid curves are strain profiles and dotted curves the damage profiles.

In agreement with the CRBS results, XRC measurements have also revealed similar differences between $\mathrm{GaAs}$ and InP target materials as well as a similar temperature effect. Figure 4 gives a few XRCs ( on the left hand side) taken from GaAs samples at two implantation doses and two different temperatures. The corresponding profiles of the perpendicular lattice strain and damage parameter (on the right hand side) are obtained by fits of the calculated XRCs to the corresponding experimental curves. The XRCs show a negative angular shift, indicating a positive strain build-up, i. e. lattice expansion, in the ion implanted region. Distribution of this strain field at low doses implantation (Fig. 4a and 4c) follows the lattice damage profiles measured by CRBS. It reaches a saturation level as the dose increases, until a uniform strain field builds up in the entire implanted layer (Fig. 4b). However, in LT implantation, the strain is subjected to better local confinement in the depth 

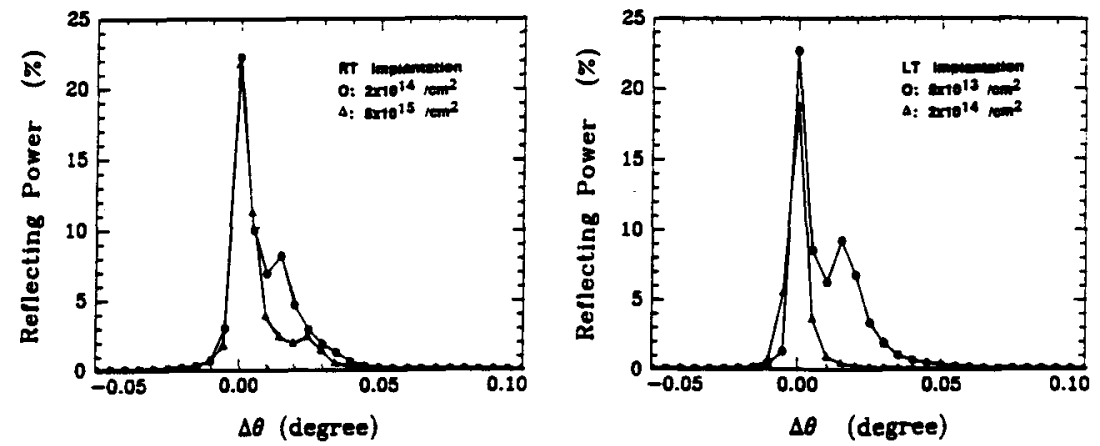

Figure 5. X-ray rocking curves of $2 \mathrm{MeV}$-oxygen-ion-implanted InP: (a) RT implanted and (b) LT implanted. Notice that the positive angular shifts are shown, indicating negative lattice strain produced in the samples.

distribution due to the "freeze-in" effect on implantation-created defects. When the damage parameter is high enough, the c-a transition takes place, where no lattice strain exhibits (Fig. 4c). No such transition can be seen in RT implantation, consistent with the CRBS results. Differences between $\mathrm{RT}$ and LT implantation are also exhibited in terms of $\mathbf{x}$-ray reflecting intensity (15\% in RT and $0.15 \%$ in LT), which is correlated with the change of the damage parameter, and the angular shift of the strain peak, which gives the magnetitude of the lattice strain $\left(0.4 \%\right.$ in RT and $0.8 \%$ in LT). More detailed analysis has been presented elsewhere ${ }^{[8]}$. Figure 5 shows a few XRCs taken from InP samples. Surprisingly, a positive angular shift is present, indicating a negative lattice strain building-up in the implanted layers. However, the magnitude of this strain field is also very small, less than $0.1 \%$. These phenomena have been observed by $\mathrm{MeV}$ nitrogen and chlorine ion implantation $[9,10]$. Comparing with GaAs, RT implanted InP samples (Fig. 5a) have features similar to the LT implanted GaAs. As the dose increases, the angular shift (i.e. the lattice strain) increases, while the intensity of the strain peak decreases, indicating that lattice damage increases and amorphization takes place. In LT implanted samples (Fig. 5b), the lattice strain builds up very quickly, even at a very low dose At a dose of $2 \times 10^{14}$ ions $/ \mathrm{cm}^{2}$, the strain peak disappears leaving a broadened, lower intensity, asymmetric substrate peak. This implies that the whole implanted layer has undergone the $c-a$ transition, in agreement with the CRBS results.

\section{DISCUSSION}

During $\mathrm{MeV}$ ion implantation, interactions between the incident energetic ions and the lattice atoms take place as the ions penetrate the sample. Near the end-of-range (EOR) of the ions, collisions between the ion and the nucleus of the lattice atoms in the low energy regime causes atomic displacements, resulting in direct structural damage to the lattice. In the surface region, interactions of the ions with electrons of lattice atoms in the high energy regime lead to massive ionization of the lattice and kinetic energy loss of the ions. Instead of direct structural damage to the lattice, electronic ionization induces thermal heating and stimulates interaction and diffusion of implantation-created lattice defects, displaced atoms and implants. We trefer to these two processes as the nuclear spike damage and the electronic spike damage. They create two distinct damaged regions as are revealed by CRBS. Thus, the lattice disordering process during $\mathrm{MeV}$ ion implantation can be classified into two primary categories: 1) defect generation and amorphization by the nuclear spike damage and 2) defect diffusion and recombination due to the associated electronic spike damage and other thermal effects. The final state of lattice disorder and the defect characteristics after implantation are the result of competition between these two processes. In III-V compound semiconductors, their binary nature makes the implantation damage process much more complicated than that in elemental semiconductors. The differences in the physical properties of two sublattice atoms (mass, atomic number, atomic size, and electronic negativity, etc.) imply that the ion-lattice interaction cross section, defect generation rate, maximum kinetic energy transfer, and range distribution of displaced atoms for each type of sublattices may be 
vastly different. The variation in lattice disorder caused by implantation is also affected by chemical binding, constitutional dissociation and atomic diffusion.

We have given a kinematic model to describe generation, recombination and diffusion of the primary point defects (interstitials, vacancies, and antisite defects) in a compound lattice target under ion implantation $[11,12]$. We find that the ion-nucleus collision is the primary factor for defect generation, and defect diffusion plays a very important role in determining the final defect population and type. In GaAs, the interaction cross sections for the two sublattices may only have small differences, since two sublattice atoms have very similar physical properties. It could be treated like a single elemental lattice. In addition, self diffusion of both III and V sublattice atoms (e.g. Ga and As interstitials) is high. As the dose increases, this diffusion would be enhanced by electronic ionization in the surface region. Thus, while these interstitials are migrating, they can either recombine with vacancies to form regular lattices and antisite defects, or diffuse towards the surface, or be trapped in amorphous zones. As a result, the lattice retains minimal structural damage due to dynanic annealing, leaving an excess of vacancies in the implanted region. This leads to an atomic density decrease and a lattice spacing expansion with build-up of a positive lattice strain which we have seen. However, in InP, In and $\mathrm{P}$ have a large mass ratio as well as great differences in ionic radius and electronic negativity[13]. These cause InP more easily disordered by ion implantation than GaAs. According to atomic collision kinetics, In has a much higher displacement cross section than that of $\mathbf{P}$, but the recoiling velocity that an In atom gains is limited due to its large mass. Thus the total number of In interstitials produced in a unit volume is greater than that of $P$ interstitials, and also the recoil In atoms are stopped easily near their original sites. As a consequence, relaxation of In interstitials and their associated defect complexes cause lattice spacing contraction, i.e. negative lattice strain.

\section{CONCLUSION}

We have observed marked differences between MeV-ion-implanted GaAs and InP in terms of lattice disordering and amophization, as well as the influence of substrate temperature. InP is shown to be more effiently disordered, with the threshold dose for the c-a transition one order of magnitude lower than that of GaAs. In RT implantation, amorphization in InP proceeds by a layer-by-layer growth. A freeze-in effect at LT makes this process even more efficient. In RT implantation into $\mathrm{GaAs}$, a strong in situ defect annealing is involved, so lattice damage is partially recoved and amorphization can not occur even at a dose of $2 \times 10^{16}$ ions $/ \mathrm{cm}^{2}$. The evidence has shown that in GaAs, there exists an excess of vacancy defects due to fast diffusion of interstitials, resulting in a lattice expansion. While in InP, In interstitials have a dominate role over vacancies, leading to weak lattice contraction.

We would like to express our gratitude to Alan Rice at Caltech for his assistance in the implantation. The work at Caltech was supported in part by NSF [DMR86-15641]. The author (F.X.) acknowledge the support from Materials Research Laboratory at Harvard University during manuscript writing and for a part of CRBS analysis.

\section{REFERENCES}

1. F. Xiong and T. A. Tombrello, Nucl. Instru. \& Meth. B40/41, 526 (1989).

2. T. A. Tombrello, J. de Physique, Col. C2, C2-1 (1989).

3. V. S. Speriosu, J. Appl. Phys. 52 (1981) 6094; and T. Vrecland Jr. and B. M. Paine, J. Vac. Sci. Technol. A4, 3153 (1986).

4. L. C. Feldman, J. W. Mayer, and S. T. Picraux, Materials Analysis by Ion Channeling (Academic Press, New York, 1982).

5. B. C. Larson and J. f. Barhoest, J. Appl. Phys. 51, 3181 (1980).

6. C. R. Wie, T. A. Tombrello, and T. Vreeland Jr., J. Appl. Phys. 59, 3743 (1986).

7. S. T. Lee, G. Braunstein, and S. Chen, Mat. Res. Soc. Symp. Proc. Vol. 126, 183 (1988).

8. F. Xiong, C. J. Tsai, T. Vreeland, T. A. Tombrello, J. Appl.Phys. (1990) (in press).

9. C. R. Wie, T. Jones, T. A. Tombrello, T. Vreeland Jr., F. Xiong, Z. Zhou, G. Bums, and F. H Dacol, Mat. Res. Soc. Symp. Proc. Vol. 74, 517 (1986).

$10 \mathrm{~F}$. Xiong, C. W. Nieh, D. N. Jamieson, T. Vreeland, and T. A. Tombrello, Vacuum (Great Britain) 39, 177 (1989).

11. F. Xiong, PhD thesis, Caltech. (Jan. 1990).

12. F. Xiong, T. Vreeland Jr., and T. A. Tombrello, to be published.

13. J. C. Phillips, Bonds and Bands in Semiconductors (Academic Press.New York. 1973), p.227. 